\title{
Numerical Simulation of Liquid Jet Atomization Including Turbulence Effects
}

\author{
Huu P. Trinh* \\ NASA-Marshall Space Flight Center, MSFC, AL 35812 \\ C. P. Chen" ${ }^{* *}$ and Balasubramanyam, M.S. ${ }^{\dagger}$ \\ University of Alabama in Huntsville, Huntsville, AL 35899
}

This paper describes numerical implementation of a newly developed hybrid model, $T$-blob/T-TAB, into an existing computational fluid dynamics (CFD) program for primary and secondary breakup simulation of liquid jet atomization. This model extend two widely used models, the Kelvin-Helmholtz (KH) instability of Reitz (blob model) and the Taylor-Analogy-Breakup (TAB) secondary droplet breakup by O'Rourke and Amsden to include turbulence effects. In the primary breakup model, the level of the turbulence effect on the liquid breakup depends on the characteristic scales and the initial flow conditions. For the secondary breakup, an additional turbulence force acted on parent drops is modeled and integrated into the TAB governing equation. Several assessment studies are presented and the results indicate that the existing $\mathrm{KH}$ and TAB models tend to under-predict the product drop size and spray angle, while the current model provides superior results when compared with the measured data.

\section{Nomenclature}

Radius of blob or parent drop

Constant (0.61)

$B_{1}$ Constant (10.0)

$\mathrm{C}_{\mathrm{a}}$ Constant $\left(\mathrm{B}_{\mathrm{o}} / 3.726 \mathrm{~B}_{1}\right)$

$\mathrm{C}_{\mathrm{b}} \quad$ Constant (1/2)

$\mathrm{C}_{d} \quad$ Discharge coefficient of injection nozzle; constant used in equation (9)

$\mathrm{C}_{\mathrm{F}} \quad$ Constant (1/3)

$\mathrm{C}_{\mathrm{jet}} \quad$ Constant (10)

$\mathrm{C}_{\mathrm{k}} \quad$ Constant (8)

$\mathrm{C}_{\mathrm{t}} \quad$ Empirical constant involving turbulence force

$c_{\text {t }} \quad$ Weighting parameter associated with turbulence motion

$c_{w} \quad$ Weighting parameter associated with surface motion

D Diameter of injection nozzle

E Term associated with energy

$k$ Turbulence kinetic energy per unit mass in the $k-\varepsilon$ model

$k_{t} \quad$ Turbulent kinetic energy

\footnotetext{
*Aerospace Engineer, Combustion Device Branch/ER32.

"Professor, Department of Chemical and Materials Engineering, AIAA Senior Member.

${ }^{\dagger}$ Graduate Research Assistant, Student Member AIAA.
} 


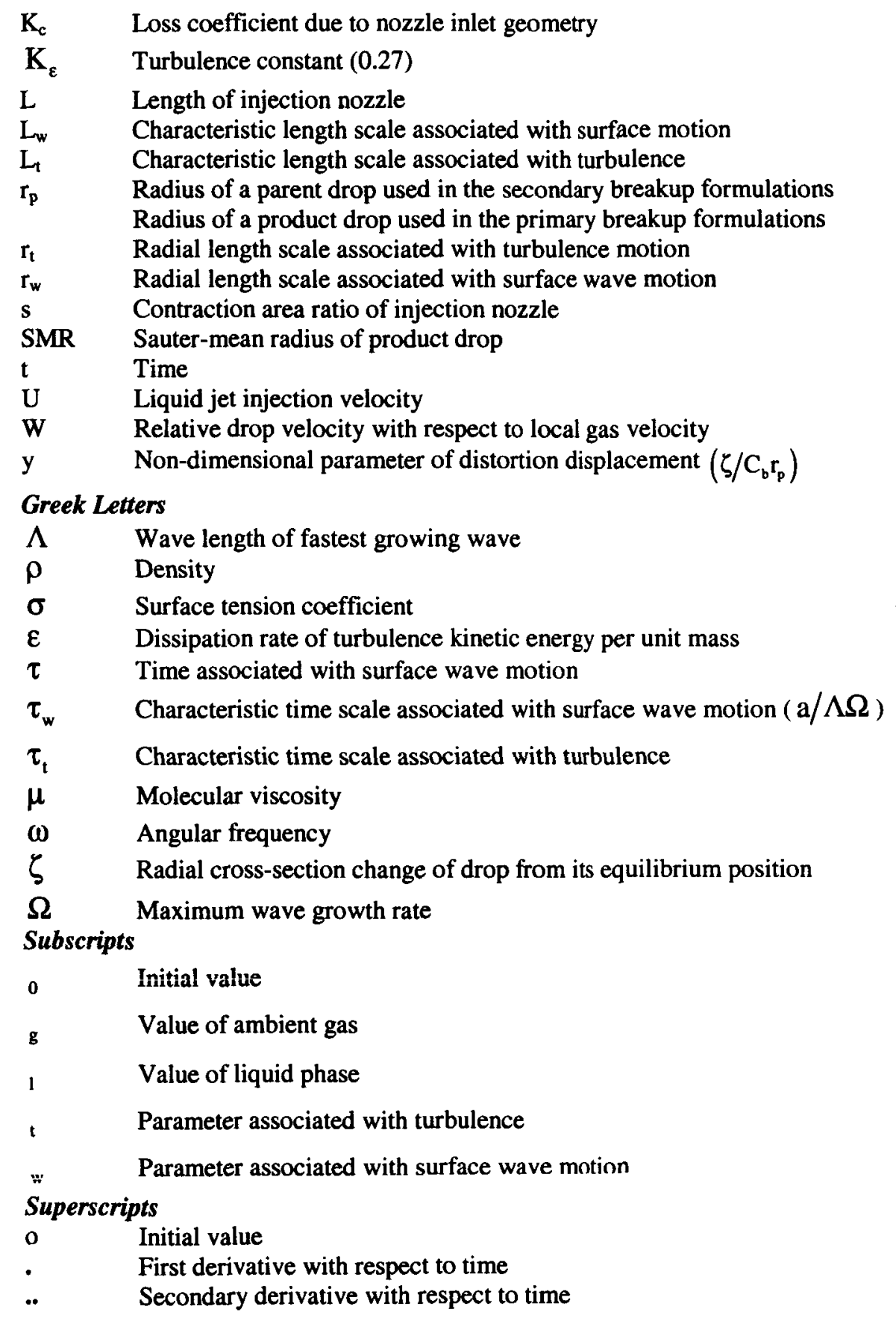

\section{INTRODUCTION}

TOMIZATION of liquid jet into a gaseous surrounding plays an important role in combustion dynamics of
conany industrial and propulsion systems. Jet inertia, aerodynamic forces, as well as the surface tension all
contributes to the jet disintegration. Often the geometrical sharpness of the injection nozzle inlet, along with
appropriate flow conditions, can create cavitations inside the nozzle. The collapse of this cavitation can generate a
flow fluctuation, leading to a more aggressive disintegration of the liquid jet. Traditional spray atomization models
such as the KH instability model of Reitz [1], the TAB of O'Rourke and Amsden [2], and some enhanced models 
[3-4], did not account for the liquid turbulence effects. Recently, Nishimura et al. [5] developed a phenomenological cavitation model in which the liquid turbulence motion within the injector was formed by the bubble collapse and the fluid turbulence motion. Their model was applied to the primary breakup regime. Considering the nozzle exit turbulence conditions of diesel sprays, Huh et al. [6] proposed a scheme taking into account two independent mechanisms, wave growth and turbulence in the atomization process. The turbulence is characterized partially by the injection nozzle geometry while the wave growth is derived from the $\mathrm{KH}$ instability theory. The aforementioned models were formulated with semi-empiricism. Several efforts of using sophisticated numerical approaches have been performed in modeling the detailed turbulent flow fields in the liquid and gas during the atomization process. In the Large Eddy Simulation (LES) spray modeling of Apte et al. [7], a stochastic approach for droplet breakup taking into a range of product-droplet sizes was developed for modeling of secondary breakup regime. De Villiers et al. [8] applied LES to resolve the primary atomization under more realistic operating conditions found in Diesel engines. These methods have a real potential of providing a complete physical description of the liquid jet breakup with minimum assumptions; however, they require submicron spatial elements in size and pico-second in time steps to properly predict the atomizing sprays at the high-velocity injection conditions. Consequently, grid mesh size and the considered physical domain must be taken care of so that the computational time and memory storage requirements can be manageable. At the present time they are still too expensive and generally impractical in terms of computational time and power requirements for engineering calculation applications. Hence, the engineering analysis and design of the liquid spray devices still must rely on phenomenological engineering models.

Based on two widely used models, Reitz's primary atomization (blob) [1] and the Taylor-Analogy-Break (TAB) [2] secondary droplet breakup, a hybrid T-blob/T-TAB model was recently developed by Trinh and Chen [9] to include turbulence effects in both primary and secondary breakup regimes. The "hybrid" model is used to explicitly differentiate between the primary and secondary breakup mechanisms that occur in different regimes. The approaches described in [5-6], and others [10-11] are hybrid models. Here, in the primary breakup model, T-blob, the level of the turbulence effect on the liquid breakup depends on the characteristic time scales and the initial flow conditions. This treatment offers a balance of contributions of liquid turbulence and perturbed surface wave physical phenomena on the liquid breakup process. For the secondary breakup, T-TAB, an additional turbulence force acted on parent drops is modeled and integrated into the $T A B$ governing equation. The drop size formed from this breakup regime is estimated based on the energy balance before and after the breakup occurrence. The turbulence energy is also considered in this process. Detailed model development and theoretical formulations, as well as several fundamental test cases and energy budget calculations were described in [9] for simplified flow fields to validate several model coefficients. The purpose of this paper is to incorporate this newly developed hybrid phenomenological T-blob/T-TAB model into a Computational Fluid Dynamics (CFD) methodology based on Eulerian (gas)-Lagrangian (liquid) formulation for complicated injector liquid-atomization and spray simulation.

\section{MODEL DESCRITION AND IMPLEMENTATION}

Detailed development and theoretical foundation of T-blob/T-TAB model was described in [9]. Only the final forms of the model are summarized here.

\section{T-blob Model}

In this model, the drop breakup rate and the mean drop size are given by:

$$
\begin{aligned}
& \frac{d a}{d t}=-\left[\frac{a}{\tau}-C_{a}\left(\frac{L_{w}}{\tau_{w}}-\frac{L_{t}}{\tau_{t}}\right)\right], \text { when } \quad r_{p}<a, \\
& r_{p}=\frac{r_{w} r_{t}}{\left(1-c_{t}\right) r_{t}+c_{t} r_{w}} .
\end{aligned}
$$


The characteristic length scale $\mathrm{L}_{w}$ and time scale $\tau_{w}$ are associated with the droplet surface wave instability. These two scales along $\tau$ and $\mathrm{C}_{\mathrm{a}}$ are formulated from the "blob" model [1] as: $\mathrm{L}_{\mathrm{w}}=\Lambda, \tau_{\mathrm{w}}=\mathrm{a} / \Lambda \Omega, \tau=3.726 \mathrm{~B}_{1} \mathrm{a} / \Lambda \Omega$ and $C_{a}=\frac{B_{0}}{3.726 B_{1}}$. The turbulence characteristic length scale $L_{t}$ and time scales $\tau_{t}$ were derived using the analytical solution of the $k-\varepsilon$ turbulence model:

$$
\begin{aligned}
& \tau_{\mathrm{t}}=\tau_{\mathrm{t}}^{0}+0.0828 \mathrm{t} \\
& \mathrm{L}_{\mathrm{t}}=\mathrm{L}_{\mathrm{t}}^{0}\left(1+\frac{0.0828 \mathrm{t}}{\tau_{\mathrm{t}}^{0}}\right)^{0.457} .
\end{aligned}
$$

The time $t$ is counted from the time at which the parent drop leaves the injection nozzle exit. The initial turbulence length scale $L_{t}^{0}$ and time scale $\tau_{t}^{0}$ are evaluated from the initial turbulent kinetic energy $k_{t}^{0}$ and its corresponding dissipation rate $\varepsilon_{\mathrm{t}}^{0}$ at the injector exit:

$$
L_{t}^{0}=c_{\mu} \frac{\left(k_{t}^{0}\right)^{3 / 2}}{\varepsilon_{t}^{0}}, \tau_{t}^{0}=c_{\mu} \frac{k_{t}^{0}}{\varepsilon_{t}^{0}} ; \text { where } c_{\mu}=0.09 \text {. }
$$

The initial turbulent kinetic energy $\mathbf{k}_{\mathrm{t}}^{0}$ and its corresponding dissipation rate $\varepsilon_{\mathrm{t}}^{0}$ can be approximated as

$$
\begin{aligned}
& k_{\mathrm{t}}^{0}=\frac{\mathrm{U}^{2}}{8 \mathrm{~L} / \mathrm{D}}\left[\frac{1}{\mathrm{C}_{\mathrm{d}}^{2}}-\mathrm{K}_{\mathrm{c}}-\left(1-\mathrm{s}^{2}\right)\right] \\
& \varepsilon_{\mathrm{t}}^{0}=\mathrm{K}_{\mathrm{e}} \frac{\mathrm{U}^{3}}{2 \mathrm{~L}}\left[\frac{1}{\mathrm{C}_{\mathrm{d}}^{2}}-\mathrm{K}_{\mathrm{c}}-\left(1-\mathrm{s}^{2}\right)\right],
\end{aligned}
$$

with $K_{e}=0.27$. The velocity $U$ is the liquid velocity at the injection nozzle, which has the length, $L$, and the diameter, D. The discharge coefficient, the loss coefficient due to the nozzle entrance sharpness, and the downstream-to-upstream contraction area ratio of the injection nozzle are represented by $C_{d}, K_{c}$, and $s$, respectively. The parent drop would no longer strip its mass to create the product drop when the parent drop radius a is less than the radius $r_{p}$ of the product droplet. In Equation (2), determination of the radius $r_{w}$ associated with the wave motion, follows the original formula of [1]; and the value of $r_{f}$, as well as weighting coefficients can be found in [9]. Along with the inclusion of the turbulence effect on the primary atomization process, the subject phenomenon also is transitioned to the secondary droplet breakup model, the T-TAB model. The required initial velocity fluctuation quantity of the product drops right after their formation was obtained by examining the energy conservation during the primary breakup process. By equating the change in the energies of the parent drop with the energy of the product drops, the turbulent kinetic energy for the product drop was derived [9] and was used to estimate the initial veiocity fluctuation for the secondary breakup process, which is discussed next.

\section{T.TAB Model}

To account for turbulence effect, the original TAB model was modified to:

$$
\ddot{y}=\frac{C_{F}}{C_{b}} \frac{\rho_{g}}{\rho_{l}} \frac{W^{2}}{r_{p}{ }^{2}}+C_{t} \frac{\varepsilon_{o}}{C_{b}{ }^{2} r_{p}{ }^{2}}\left[\frac{k}{k_{o}}\right]^{C_{e}} \dot{y}^{-1}-C_{k} \frac{\sigma}{\rho_{l} r_{p}{ }^{3}} y-C_{d} \frac{\mu_{1}}{\rho_{l} r_{p}{ }^{2}} \dot{y}
$$

Except for the second term on the right hand side, equation (7) is the same as the governing equation of the TAB model. Again, reference [9] should be consulted for detailed derivation of this model. To estimate the post-breakup drop size, a similar method as used in the TAB model is employed for the calculation of Sauter mean radius (SMR):

$$
\operatorname{SMR}=\frac{4 \pi r_{p}^{3} \sigma}{E_{p x t}-\frac{\pi}{6} r_{p}^{5} \rho_{1} \dot{y}^{2}} .
$$


Based on the conservation principle, the initial turbulent kinetic energy $\left(k_{0}\right)$ and dissipation rate $\left(\varepsilon_{0}\right)$, in Equation (7), of the new drop were formulated [9]. $E_{\text {par }}$ in Equation (8) is the total energy of the original drop prior to the breakup and is determined from the original TAB model.

\section{T-blob Model Implementation}

The liquid is assumedly injected in a form of "blob" parcels containing spherical drops with their initial size equal to the nozzle exit dimension. The computational approach for the primary atomization proposed by Reitz [1] is adopted in the present study. Extra relations representing the turbulence are described here. The initial turbulence quantities are estimated based on Equations (5) and (6). Values of the turbulence characteristic scales are then calculated from these turbulence values. Individual parcel is tracked at each time step, and change in "blob" drop size is also determined using Equation (1). Next, the mass stripped from the parent drops is computed and is formed into a new product drop parcel. The primary breakup of the blob drops continues until their size reduces to the dimension of the product drops.

It is not necessary to create a new parcel in each computational time step. For the purpose of saving computer memory and computational time, the stripped mass would be accumulated until certain criteria are met. Then, a new parcel is added to the computations and its drop size is calculated from Equation (2). The criterion of forming a new parcel varies among authors. For instance, Reitz [1] defined the creation of a new parcel when the accumulated mass had exceeded $3 \%$ of the original parcel mass; while Huh et al. [6] used the value of $10 \%$. It was found that at least for the test cases of interest, the computational results from theses two criteria are not much different, although the number of parcels in the computational domain varies considerably. In CFD-ACE+ [13], a new parcel is created when the number of product drops is greater than $20 \%$ of the ones in the parent. In the present study, the initial turbulent kinetic energy of the product drops is estimated from the energy conservation formulation described in [9]. When this estimated energy is less than $0.1 \%$ of the kinetic energy of the droplet motion, it is assumed that the turbulence motion within the droplet becomes insignificant. Then, no turbulence is considered in the secondary breakup for subject droplet, and the classical TAB model is employed. Otherwise, the drops would be considered for the secondary breakup with the turbulence effects that are described in the next subsection.

\section{T-TAB Model Implementation}

While the parcels produced from the primary atomization are continuously tracked in the Lagrangian coordinate system, they are also considered for the secondary breakup, which is governed by Equation (7). This equation is solved in the same way as the classical TAB model [2] but using the finite difference method since its exact analytical solution does not exist.

To develop the finite difference formulation for Equation (7), it is convenient to define a new set of coefficients for this equation as follows:

$$
C_{1}=\frac{C_{F}}{C_{b}} \frac{\rho_{g}}{\rho_{1}} \frac{W^{2}}{r_{p}^{2}} \quad C_{2}=C_{t} \frac{\varepsilon_{o}}{C_{b}^{2} r_{p}^{2}}\left[\frac{k}{k_{b}}\right]^{C_{1}} \quad C_{3}=C_{k} \frac{\sigma}{\rho_{1} r_{p}^{3}} \quad C_{4}=C_{d} \frac{\mu_{1}}{\rho_{1} r_{p}^{2}},
$$

By substituting the above coefficients, Equation ( 8 ) becomes

$$
\ddot{y}=C_{1}+C_{2} \dot{y}^{-1}-C_{3} y-C_{4} \dot{y}
$$

In contrast to the $\mathrm{TAB}$ model, an exact solution can not be obtained for since it contains an extra non-linear term (second term on the RHS). Instead, a second order finite central difference scheme in a time step was used [12]. The deformation displacement $y$ is computed at every time step. The secondary breakup assumedly occurs when $y$ approaches 1. Then the product drop size is calculated from Equation (8). At the same time, its initial turbulence quantities are estimated [9]. Again, the new product drops are continuously tracked for possible future secondary atomization until they leave the computational domain. When the product drop size is smaller than the Kolmogorov length scale, the turbulence activity of the new drop is no longer considered. In this case, the present model would recover the classical $\mathrm{TAB}$ model.

The T-blob/T-TAB model was incorporated into the CFD-ACE+ [13] program for two-way gas-liquid atomization calculations. This code, together with its liquid spray module, solves the equations of steady-state as well as transient flow with chemically reactive fluid dynamics and droplet spray. The governing equations and the 
numerical solution method are discussed in [13] and are only described briefly here. The Navier-Stokes equations and RNG (Renormalized Group) $k-\varepsilon$ turbulence model for a gas phase are solved with a finite volume method. A time-marching with a high-order accurate explicit scheme is employed in the transient calculation. With an assumption of dilute spray, parcels of drops are tracked in a Lagrangian framework. The parcel represents an ensemble of identical drops occupied in the same space at a given time. Subsequently, the spray properties at each point are described by statistically sampling the spray parcels. The drop parcels exchange mass, momentum, and energy with the gas through source terms in the Navier-Stokes equations.

\section{RESULTS and DISCUSSION}

Numerical simulations of several test cases as listed in Table 1 are performed and compared with available measured data. Variations in the predictions from the two classical $\mathrm{KH}$ and TAB models and the present models are also examined. The two-dimensional axisymmetric flow of a cylindrical liquid jet located at the center line of the computational domain is simulated. It should be noted that the current version of CFD-ACE+ does not have collision/coalescence models. Due to the observation of these phenomena [15], a module was written [12] to compute the droplet collision and coalescence based on model of [14]. The time-transient liquid jet breakup and the ambient gas behavior are computed for an incremental time step, $\Delta \mathrm{t}$, of $2.5 \times 10^{-6} \mathrm{sec}$. The spray trajectory is tracked in the Lagrangian coordinate system. A different time step, which is normally smaller, is applied for this coordinate system. CFD-ACE+ recalculates this time step continuously based on criteria of pre-assigned maximum allowable changes in drop velocity and diameter. The Lagrangian tracking time step used in the simulations for this study ranges from $1 / 3$ to $1 / 10$ of $\Delta t$. It should be noted that a solution sensitivity study for various numerical parameters, such as grid mesh size, time step values, etc. has been performed [12] prior to obtaining the results of this investigation.

Table 1. Test cases and measured data used in the computations

\begin{tabular}{|c|c|c|c|c|}
\hline Case & $\mathrm{H}-1$ & $\mathrm{H}-2$ & $\mathrm{H}-3$ & $\mathrm{~K}$ \\
\hline Nozzle Diameter (mm) & & 0.3 & & 0.24 \\
\hline Ambient Gas & & Nitrogen & & Nitrogen \\
\hline Ambient Pressure (MPa) & 1.1 & 3.0 & 5.0 & 2.17 \\
\hline Ambient Temperature (K) & & 298 & & 298 \\
\hline Density $\left(\mathrm{kg} / \mathrm{m}^{3}\right)$ & 12.36 & 33.70 & 56.17 & 24.51 \\
\hline Liquid Fuel & & Diesel Fuel & & Diesel Fuel \\
\hline Density ( $\left(\mathrm{kg} / \mathrm{m}^{3}\right)$ & & $\hat{8} \mathbf{4 0}$ & & 840 \\
\hline Viscosity $\left(\mathrm{Kg} / \mathrm{m}^{\prime} \mathrm{s}\right)$ & & $2.9 \times 10^{-3}$ & & $5.0 \times 10^{-3}$ \\
\hline Surface Tension (N/m) & & $2.05 \times 10^{-2}$ & & $2.06 \times 10^{-2}$ \\
\hline Injection Velocity $(\mathrm{m} / \mathrm{s})$ & 102.0 & 90.3 & 86.41 & 133.81 \\
\hline \multicolumn{5}{|l|}{ Initial Turbulence Quantity } \\
\hline Kinetic Energy $\left(\mathrm{m}^{2} / \mathrm{s}^{2}\right)$ & $2.88 \times 10^{2}$ & $2.26 \times 10^{2}$ & $3.94 \times 10^{1}$ & $5.22 \times 10^{2}$ \\
\hline $\begin{array}{l}\text { Dissipation Rate of Kinetic Energy } \\
\left(\mathrm{m}^{2} / \mathrm{s}^{3}\right)\end{array}$ & $1.06 \times 10^{8}$ & $7.34 \times 10^{7}$ & $1.22 \times 10^{7}$ & $3.14 \times 10^{8}$ \\
\hline Reference & \multicolumn{3}{|c|}{ Hiroyasu et al. [15] } & Koo [16] \\
\hline
\end{tabular}

The spray tip penetrations from the exit port into the ambient gas for test cases $\mathrm{H}-1$ and $\mathrm{H}-2$ are plotted in Figure 1 as a function of time. Both predictions from the T-blob/T-TAB and KH/TAB are similar. Nearly straight lines of 
the tip penetration curves at the beginning indicate that the tip velocity of the liquid jets remains almost constant. Once the jet is fully converted into droplets, the surface area of the spray nose is enlarged, and its speed is subsequently reduced due to greater aerodynamic force and drag. Hence, the penetration curve has a smaller slope at the later time. Furthermore, the results also reveal that the spray penetrates at faster rate into a lower pressure gas. In comparison with measurements, the tip penetrations in both cases are slightly under-predicted with analytical models at the beginning of the spray; however, they are over-predicted somewhat at the later time. Overall, the computational results show a close agreement with the experimental data. Variations in the spray angle due to different ambient gas pressures for test cases $\mathrm{H}-1, \mathrm{H}-2$, and $\mathrm{H}-3$ are depicted in Figure 2. This angle is a global parameter used to characterize the radial expansion of the spray. For an axisymmetric case this angle, as shown in Figure 2, is formed by the two opposite tangent lines drawn along the spray envelop. The results portray higher

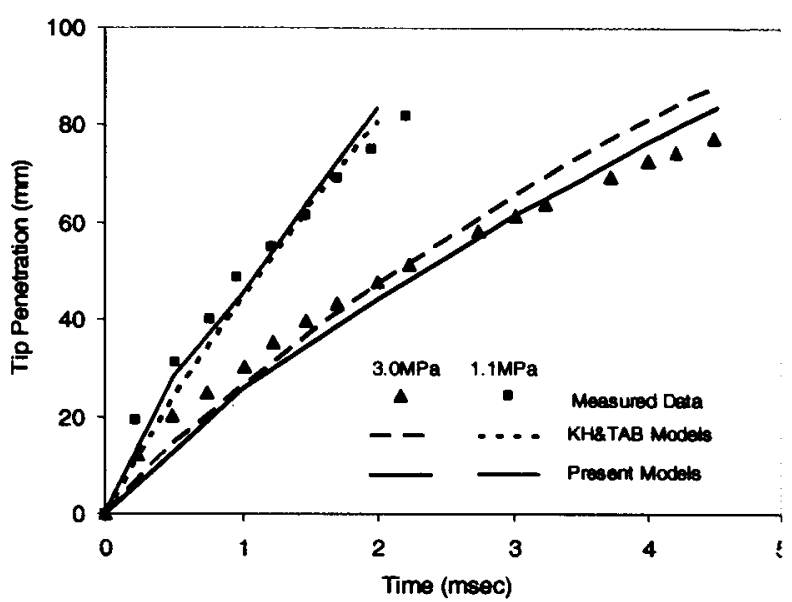

Figure 1. Comparison of tip penetration.

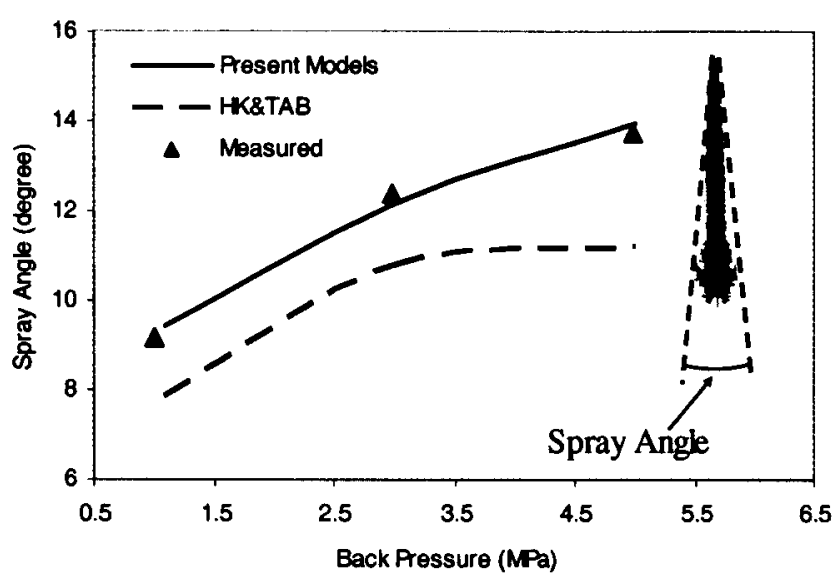

Figure 2. Variations in spray angles vs. back $P$

back pressure with a larger radial expansion of the spray. A further examination of the atomization modeling suggests that the radial product drop velocity is related to the wave length and its maximum growth rate. The values of these parameters, however, are dependent on the liquid-to-gas density ratio. Consequently, higher gas pressure would result in a larger radial-to-axial velocity ratio and a wider spray angle. In comparison with the results of the $\mathrm{KH} / \mathrm{TAB}$ models, a favorable larger spray angle is predicted by the present models. As stated in [9], the T-blob model estimates considerably larger product drop sizes than the $\mathrm{KH}$ model, which leads to the prediction of a stronger radial momentum. Consequently, liquid drops are spread outward more in the T-blob model when compared to the predictions provided by the $\mathrm{KH}$ models. As shown in Figure 2, a better agreement with the measured data is obtained with the present models.

The computed spray shapes and tip penetrations from the present models are displayed in Figure 3 for three different back pressure conditions after $2.5 \mathrm{~ms}$ of liquid injection. It should be noted that the injection velocity is higher for a lower back pressure condition. Hence, the spray tip penetrates faster into a lower pressure environment, although the drag increases on the periphery of the spray contour. This phenomenon leads to parcels containing small drops being dragged into the wake behind the tip region, which clearly can be observed in Figure 3 (a) and (b). It is also interesting to note that larger drops appear on the spray tip as well as on the peripheral regions near the tip. Generally, large drops carry a stronger momentum, which decays less than their smaller drop counterparts. Hence, the large drops tend to travel faster downstream than the smaller drops do. In addition, large drops found in these regions may reflect the effect of drop coalescences. The coalescence is more pronounced in an elevated gas pressure. As illustrated in Figure 3(c), a larger drop size can be observed in the tip region at higher ambient pressures.

The following results are collected from numerical simulations of test case $K$ (see Table 1). This test case is selected to evaluate the atomization models because of its available measured drop size data. In the experiment, Koo [16] measured diesel sprays with a Phase/Doppler Particle Analyzer (PDPA) system. In general, a PDPA can recognize only a defined range of droplet diameters due to digital processing hardware limitations. Normally, the 
ratio of the largest observable droplet to the smallest observable droplet cannot exceed 35 . Regarding to this experiment, the instrument was set to measure drop sizes from 1.8 to $246 \mu \mathrm{m}$. It should be noted that the liquid fuel injection was driven by a cyclic pumping system for which the mass injection rate is no longer constant. To simulate more realistically the inlet flow conditions, the transient injection velocity profile reported by Koo [16], as shown in Figure 4, is utilized in the computations.

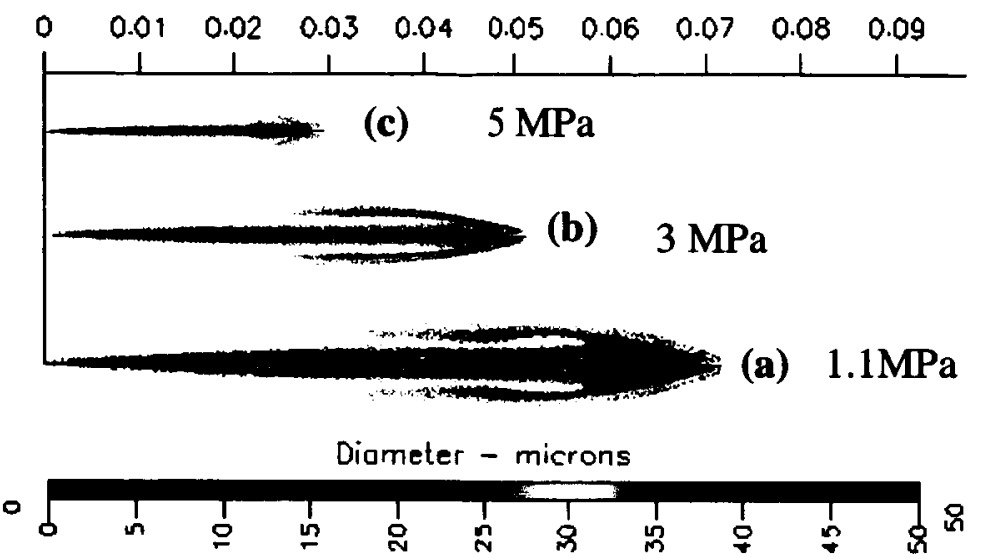

Figure 3. Variations in spray shape, tip penetration, and drop size at $t=2.5 \mathrm{~ms}$ due to different back pressures,

(a) $1.1 \mathrm{MPa}$; (b) $3 \mathrm{MPa}$; (c) $5 \mathrm{MPa}$

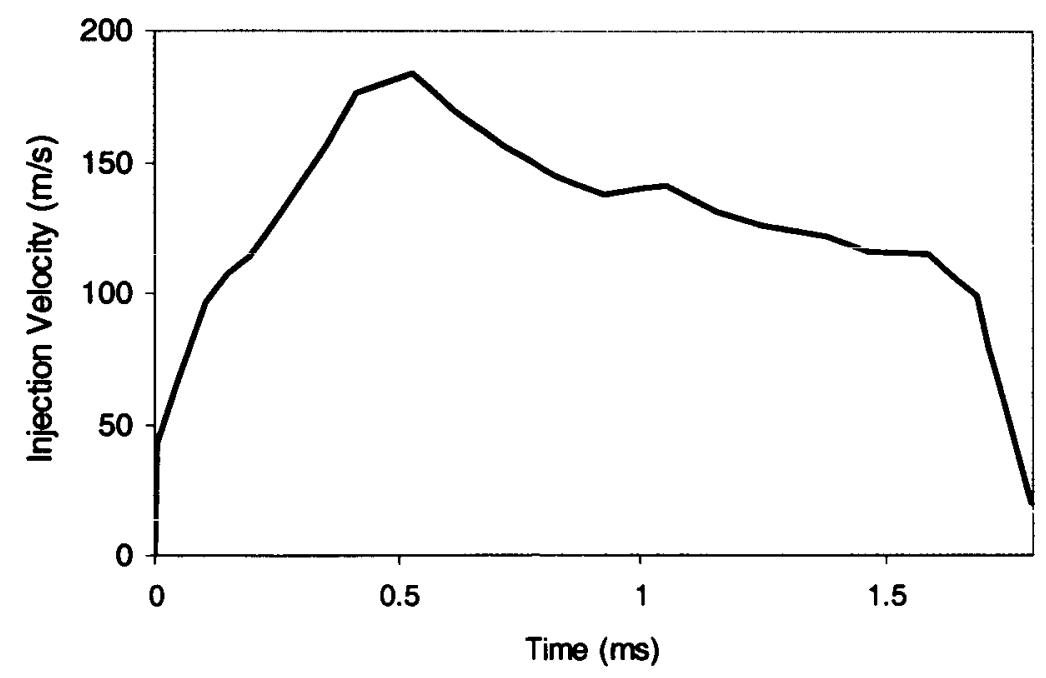

Figure 4. Fuel injection velocity used in simulation for test case $\mathrm{K}$

Similar to the previous computational cases, this test case is simulated also for the transient flow field conditions with $\Delta t=2.5 \times 10^{-6} \mathrm{~s}$. It should be noted that the present simulation utilizes the RANS approach to obtain the mean velocity field of the gas phase. For statistically non-stationary flows, which were investigated experimentally by Koo [16] in this case, the interpretation of the averaging process is the ensemble averaging; thus the RANS formulation is justified for the statistically transient case as indicated by the injector inlet condition of Figure 4 . The predicted spray tip penetration and the measured data are presented in Figure 5. Also shown in this figure are the grid-refinement comparative results. With finer grids packed in the near nozzle region, the spray seemed to be "denser" and penetrate further into the gaseous environment. This is the general shortcoming of Eulerian-Lagrangian approach utilizing particulate "point source" assumption within the Eulerian grids [17]. Extremely fine grids can not 
be used. Within certain grid density, the spray exhibits similar behavior. The predictions of this test case, made with both the $\mathrm{KH} / \mathrm{TAB}$ and present models, are slightly lower than the measurement at the beginning of the injection; however, the predicted curves approach the experimental data at a later time. It is noted that Koo reported the spray penetration length from the beginning of the injection to the time of $1.5 \mathrm{~ms}$; while the computation continues to 3.0ms. In general, both the predicted tip penetrations are similar, and their values are in reasonable agreement with the experimental data. Figure 6 displays the simulated spray shapes along with the photographs recorded at several time intervals in the experiment. In comparison with the photographs, the simulated spray penetrates with a slightly shorter distance into the ambient gas than the actual distance depicted from the photographs initially; however, the

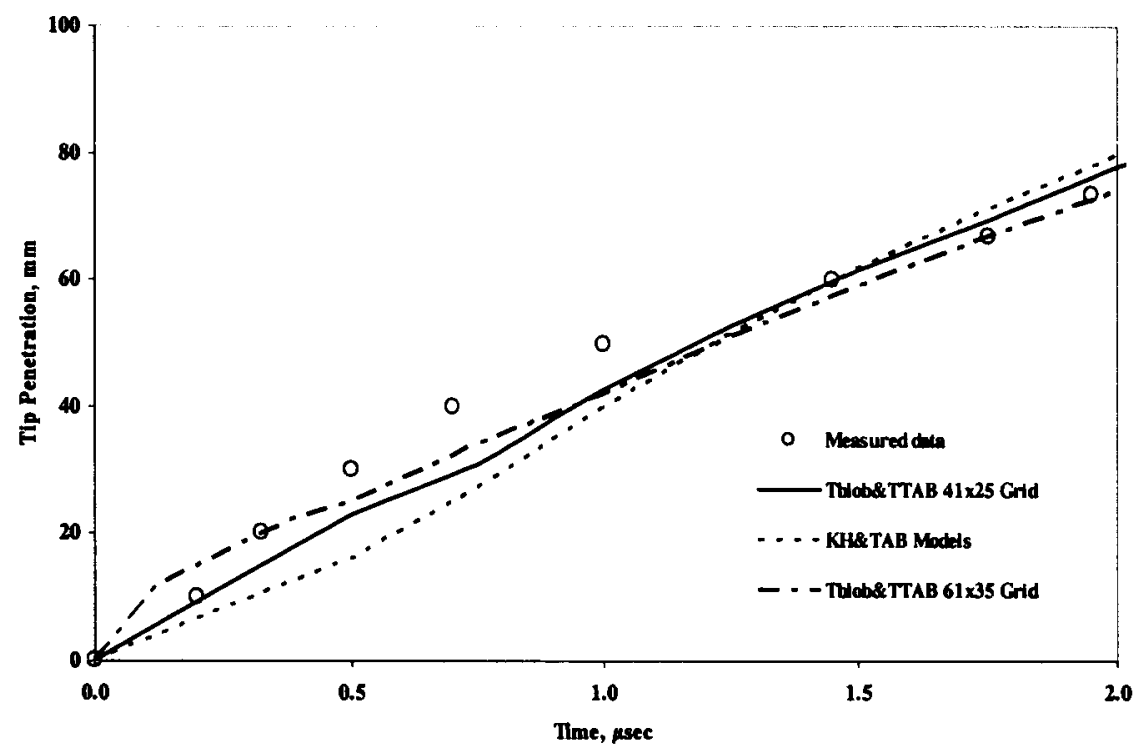

Figure 5. Predicted and measured spray tip penetration for test case $\mathbf{K}$

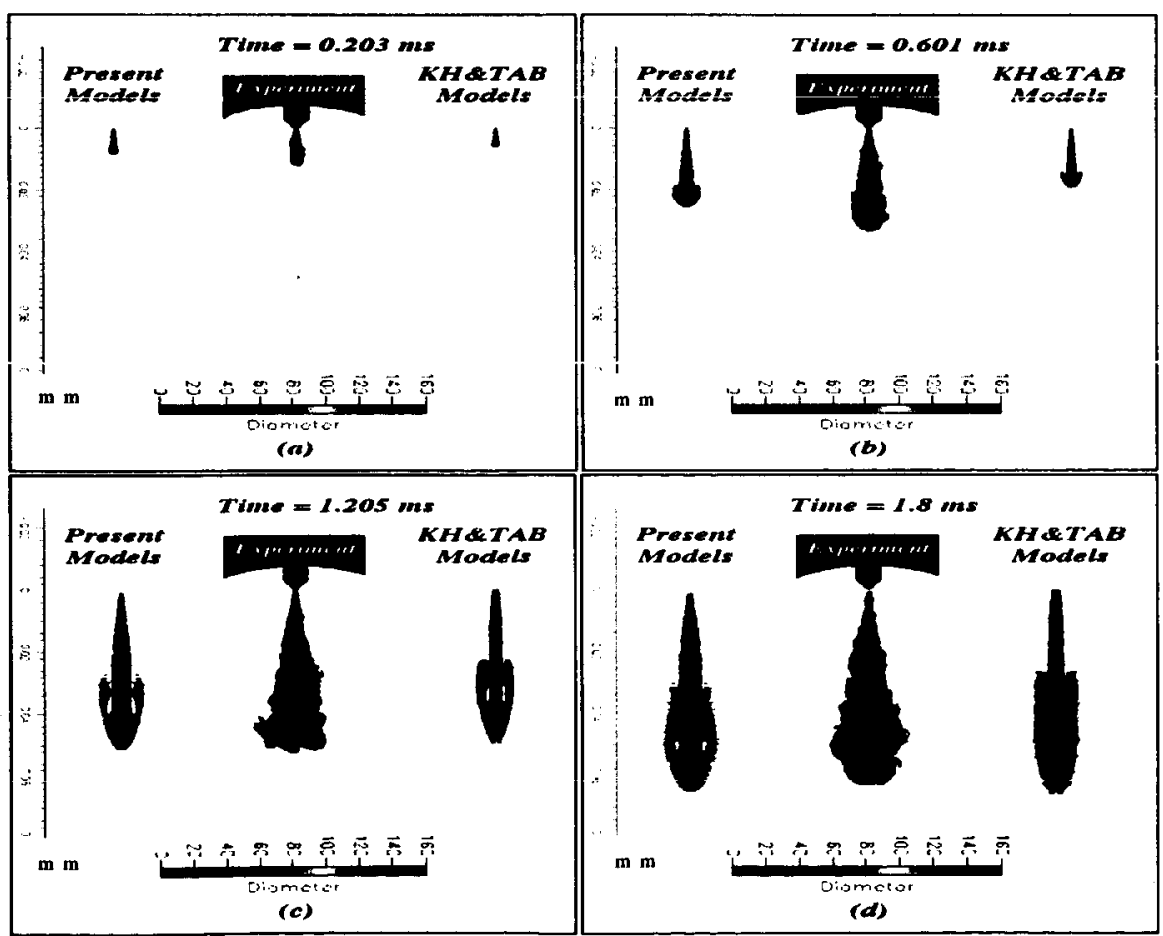

Figure 6. Simulations and photographs of case K; (a) $\mathrm{t}=0.203 \mathrm{~ms}$; (b) $0.601 \mathrm{~ms}$;(c) $1.205 \mathrm{~ms}$;(d) $1.8 \mathrm{~ms}$ 


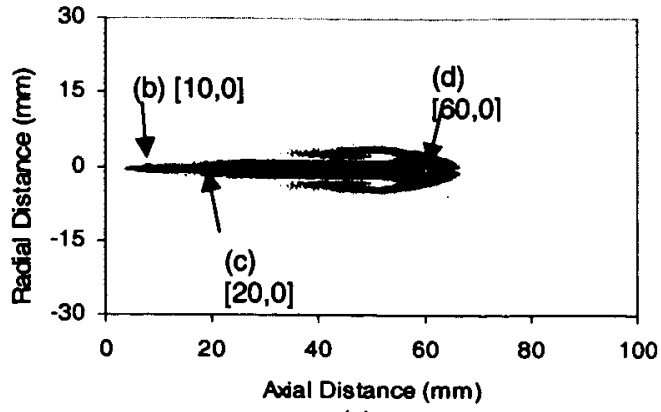

(a)

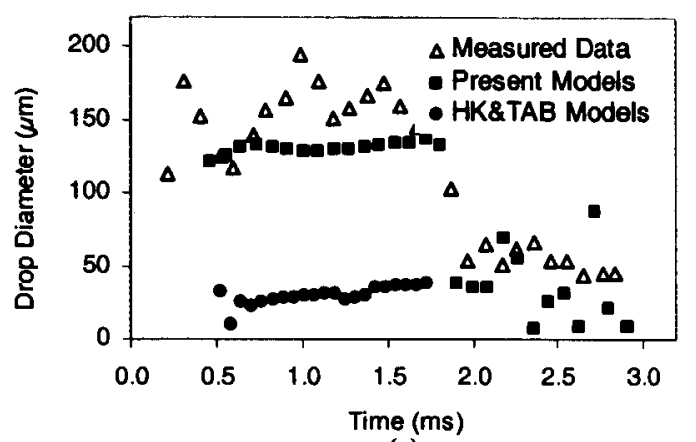

(c)

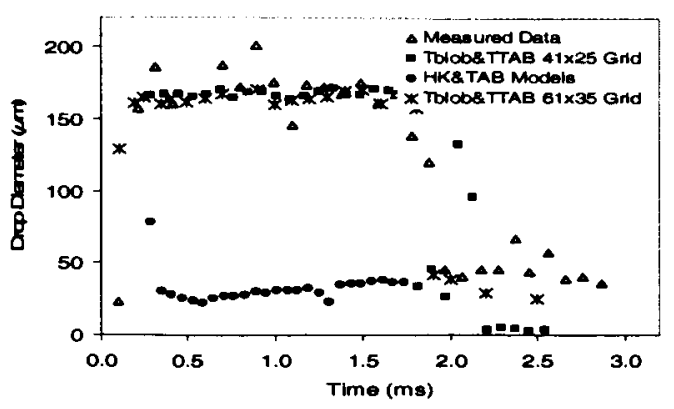

(b)

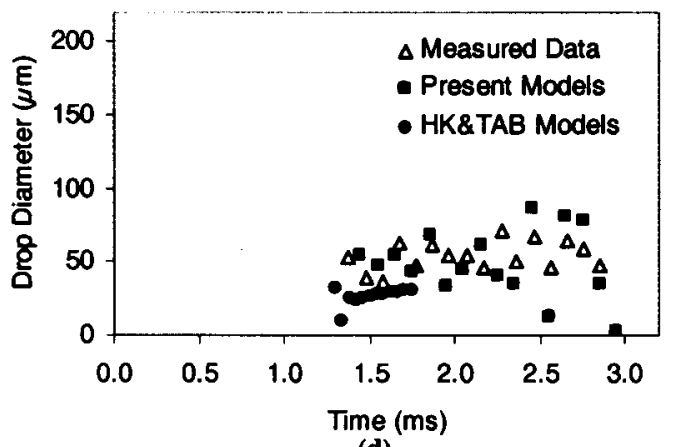

(d)

Figure 7. Drop size distributions from the predictions and measurements for test case $\mathrm{K}$

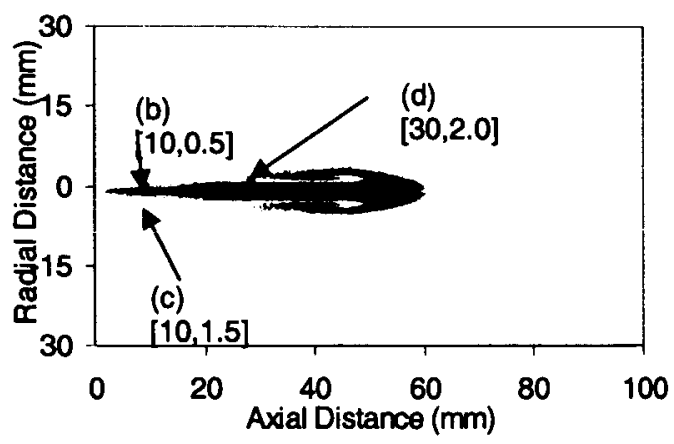

(a)

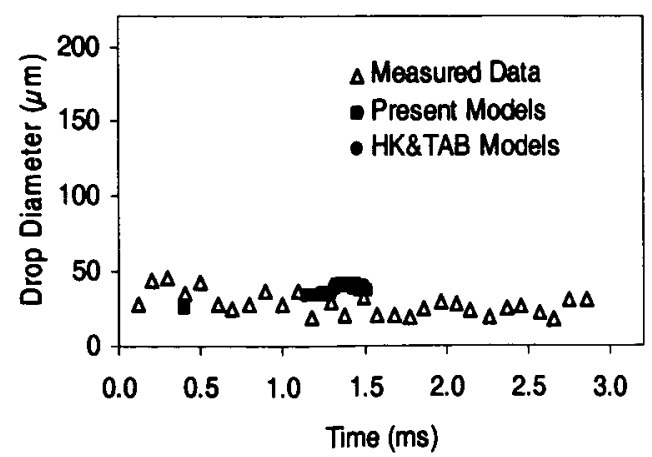

(c)

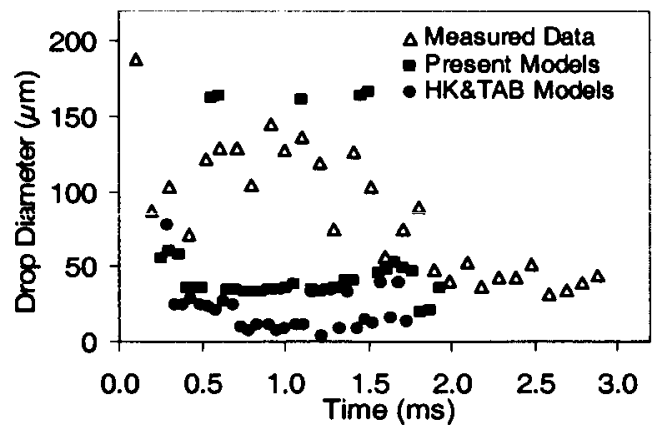

(b)

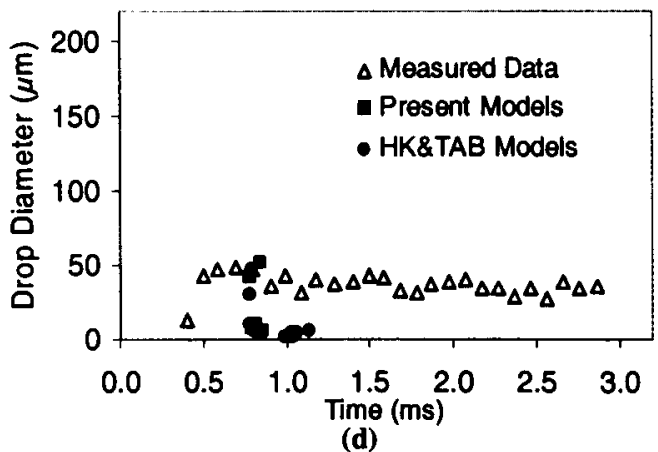

(d)

Figure 8. Drop size distributions from the predictions and measurements for test case $\mathrm{K}$ 
predictions improve significantly afterwards. These results also reflect the same assessment of the tip penetration comparison shown in the previous Figure 5. It is also interesting to observe the degree of the spray being spread laterally and its tip shape. All the models predict a similar radial expansion of the spray for all considered time intervals, and they are compared reasonably well with their photographic counterparts. However, the numerical simulations display the tip profile more like a conical shape with parcels containing large drops rather than a round tip shown in the photographs. Since the momentum of these large drops is degrading much less than the one of the small drops, the large drops move faster downstream than the smaller drops. Therefore, the moving motion of the large drops creates the appearance of the conical shape at the tip. A series of the computational cases has been conducted to examine the tip shape variation for different initial spray angles. The analysis suggests that the spray tip head-end changes to a round-shape and does not penetrate into the ambient gas as deep when increasing the initial spray angle. The tip variation analysis is not addressed in detail here, since it is not a main focus in this study.

Further detailed drop size comparisons between the predictions and the measurements are presented in Figures 7 and 8. Figure 7 reveals the drop sizes recorded at three axial locations along the center line of the spray, which are identified in Figure 7(a). The results indicate that the jet, with an initial diameter of $240 \mu \mathrm{m}$, breaks up into relatively large drops along the center line. Their product drop diameters range from 100 to $200 \mu \mathrm{m}$ at the stations of $x=10$ and $20 \mathrm{~mm}$ (Figures 7(b) and (c)). These results suggest that the primary breakup process is dominant in these regions. Further downstream, the drops undergo the secondary atomization which generates smaller drops. Figure 7(d) exhibits drops of 20 to $50 \mu \mathrm{m}$ in diameter at the $x=60 \mathrm{~mm}$ station. It should also be noted that the fuel injection cycle for this test case is complete at $1.77 \mathrm{~ms}$; therefore, the drop sizes shown in Figures 7 (b) and (c) also reflect this end period at which the small drops are registered. In Figure 7(b), the effect of finer grid within this region is also observed. As discussed earlier, the finer grid system seems to see "more" droplets even the spray has been terminated (after $2 \mathrm{~ms}$ ). The small drops recorded after $1.77 \mathrm{~ms}$ are formed because of the small injection rate at the tail end of the injection cycle, as well as the small drops lagging behind in the spray motion. In general, the predictions from the present models are in good agreement with the measured data. In contrast, the HK and TAB models under-predict the drop size for stations of $x=10$ and $20 \mathrm{~mm}$; however, their predictions are improved significantly at the $x=60 \mathrm{~mm}$ station. This observation is consistent with earlier results in [9]. The results also show that the KH model predicts generally smaller drop sizes than the T-blob model. Figure 8 displays the drop sizes at three off-centerline locations $[x, y]$ shown in Figure 8(a). For the location at $[10,0.5]$, the measurement registered relatively large drops, the diameters of which are ranging from 30 to $180 \mu \mathrm{m}$ with a random order in time. Similar to the results shown in the previous figure, the smaller drops are also observed after the end of the injection cycle at $t=1.77 \mathrm{~ms}$. The present model predictions capture large drops intermittently at certain short time intervals; however, both the sets of the KH/TAB and T-blob/T-TAB atomization models project typically a much smaller drop size than the measurement portrayed. In other words, the CFD simulation indicates that the secondary droplet breakup process takes place at this location but not the primary breakup. The drop diameter at $[10,1.5]$ and $[30,2]$ in Figures 8(c) and 8(d), respectively, varies from 20 to $50 \mu \mathrm{m}$. This suggests that considerably smaller drops appear on the spray periphery and their presence in this outermost region forms the shape of the spray. Furthermore, since the drop sizes at these locations are relatively small, the secondary droplet breakup occurs mostly in these regions. This assessment is consistent with the observation of Koo in his measured data analysis. He concluded that the small droplets in the periphery of the spray are not those directly injected from the nozzle. The small droplets from the nozzle travel much slower than the large droplets, which are produced in the primary breakup; hence, they can not maintain the spray shape observed in the experiments without the appearance of drops formed by the secondary breakup mechanism. It is interesting to note that the simulation with the $\mathrm{KH}$ and TAB models has a limited number of parcels and no parcel tracked at [30,2] and [10,1.5], as shown in Figures 8(c) and 8(d), respectively. Again, this examination reconfirms the display in Figure 2 that the spray angle predicted by the classical KH and TAB models is a little smaller than the actual angle. In the present model, the numerical simulation captures droplets at $[10,1.5]$ and $[30,2]$ only in a short time span, and the predicted drop sizes at this interval are close to the measured data.

\section{CONCLUSIONS}

We have successfully implemented a newly developed hybrid T-blob/T-TAB atomization model into an existing CDA-ACE+ code for simulation of liquid jet atomization using two-way Eulerian-Lagrangian method. A drop collision and coalescence model [14] has also been incorporated into the ACE+ code to provide more realistic simulations. Assessments of the new model have been performed against two experimental studies and several observations and conclusions can be drawn as follows: 
1. In general, the CFD simulations are able to capture similar spray structures observed in the experiments. The spray tip penetration lengths as well as the appearances of large drops at the spray tips, which are predicted from the two sets of the KH/TAB and T-blob/T-TAB atomization models, are comparable to the measured data. However, the $\mathrm{KH}$ and TAB models estimate smaller spray angles for the test cases $\mathrm{H}-1, \mathrm{H}-$ 2 , and $\mathrm{H}-3$ than the measurements indicate.

2. For the test case $\mathrm{K}$, the drop measurements and the predictions indicate that small drops created from the secondary breakup appear in the outermost regions of the spray. Hence, this breakup mechanism is responsible for the radial expansion of the spray. These drops then form the profile of the spray. On the other hand, the drops on center line of the spray are relatively large. Furthermore, the appearance of large drops at the spray tips may result from drop coalescence. In addition, the strong momentum of the large drops allows them to travel ahead downstream.

3. In summary, for test case $\mathrm{K}$, the CFD simulation using the HK and TAB models tends to under-predict the drop size of the spray, while it is able to capture reasonably the liquid spray structure. On the other hand, the present models predict comparable drop sizes, as seen in the measured data. Overall, the predicted results from the present models do agree reasonably with the experimental data.

\section{ACKNOWLEDGMENTS}

This research is supported by NASA Marshall Space Flight Center in Huntsville, Alabama under a full time study program. Technical consultations of Dr. D.S. Crocker, Dr. B. Zuo, and Dr. S. Kim of CFD Research Corporations in this study are acknowledged. Finally, helpful assistance of librarians at Redstone Scientific Information Center in Huntsville, Alabama is appreciated.

\section{REFERENCES}

1. Reitz R.D., "Modeling Atomization Processes in High-Pressure Vaporizing Sprays," Atomization and Spray Technology 3, 1987, pp. 309-337.

2. O'Rourke P. J., Amsden, A. A., "The Tab Method for Numerical Calculation of Spray Droplet Breakup," SAE Technical Paper 872089, 1987.

3. Tanner, F.X., "Liquid Jet Atomization and Drop Breakup Modeling of Non-Evaporation Diese! Fuel Sprays," SAE 1997 Transactions: Journal of Engines, Vol. 106, Sec. 3, 1998, pp. 127-140.

4. Tanner, F.X., "A Cascade Atomization and Drop Breakup Model for the Simulation of High-Pressure Liquid Jets," SAE paper 2003-01-1044.

5. Nishimura, A., Assanis, D. N., “A Model for Primary Atomization Based on Cavitation Bubble Collapse Energy,” ICLASS 2000, Pasadena, CA, July 16-20, 2000.

6. Huh K.Y., Lee E., Koo J.Y., "Diesel Spray Atomization Model Considering Nozzle Exit Turbulence Conditions," Atomization and Spray, Vol. 8, 1998, pp. 453-469.

7. Apte, S. V., Gorokhovski, M. and Moin, P., "LES of Atomizing Spray with Stochastic Modeling of Secondary Breakup," Int. J. Multiphase Flow, Vol. 29, 2003, pp. 1503-1522.

8. De Villiers, E., Gosman, A.D., Weller, H.G., "Large Eddy Simulation of Primary Diesel Spray Atomization," 2004-010100, 2004 SAE International.

9. Trinh, H. P. and Chen, C. P., "Development of Liquid Jet Atomization and Breakup Models Including Turbulence Effects," Atomization and Spray, to appear; also see AIAA paper 2005-0154, 2005.

10. Bianchi, G. M. and Pelloni, P., "Model the Diesel Fuel Spray Breakup by Using a Hybrid Model," SAE Paper 1999-010226, 1999.

11. Arcoumanis, C. and Gavaises, M., "Linking Nozzle Flow with Spray Characteristics in a Diesel Fuel injection System," Atomization and Spray, Vol. 8, 1998, pp. 307-347.

12. Trinh, H. P., "Modeling of Turbulence Effect on Liquid Jet Atomization," Ph.D. Dissertation, University of Alabama in Huntsville, 2004.

13. CFD-ACE+ Theory Manual, CFD Research Corporation, Huntsville, Alabama, 2003.

14. O'Rourke, P. J. and Bracco, F. V., "Modeling of Drop Interactions in Thick Spray and Comparision with Experiments," Inst. Mech. Engrs, 1980, pp. 101-106.

15. Hiroyasu, H., Kadota, T., "Fuel Droplet Size Distribution in Diesel Combustion Chamber," SAE Paper 740715, 1974.

16. Koo, J., "Characteristics of a Transient Diesel Fuel Spray," Ph. D. Dissertation, University of Wisconsin-Madison, 1991.

17. Salman, H. and Soteriou, M., "Lagrangian Simulation of Evaporating Droplet Sprays," Physics of Fluids, Vol. 16, 2004, pp. 4601-4622. 